The University of Southern Mississippi The Aquila Digital Community

Faculty Publications

$11-28-2017$

\title{
Attractiveness as a Function of Skin Tone and Facial Features: Evidence From Categorization Studies
}

Elena V. Stepanova

University of Southern Mississippi, elena.stepanova@usm.edu

Michael J. Strube

Washington University

Follow this and additional works at: https://aquila.usm.edu/fac_pubs

Part of the Psychology Commons

\section{Recommended Citation}

Stepanova, E. V., Strube, M. J. (2017). Attractiveness as a Function of Skin Tone and Facial Features: Evidence From Categorization Studies. Journal of General Psychology (1), 1-20.

Available at: https://aquila.usm.edu/fac_pubs/16569

This Article is brought to you for free and open access by The Aquila Digital Community. It has been accepted for inclusion in Faculty Publications by an authorized administrator of The Aquila Digital Community. For more information, please contact Joshua.Cromwell@usm.edu. 
Attractiveness as a Function of Skin Tone and Facial Features: Evidence from Categorization Studies 


\begin{abstract}
Participants rated the attractiveness and racial typicality of male faces varying in their facial features from Afrocentric to Eurocentric and in skin tone from dark to light in two experiments. Experiment 1 provided evidence that facial features and skin tone have an interactive effect on perceptions of attractiveness and mixed-race faces are perceived as more attractive than singlerace faces. Experiment 2 further confirmed that faces with medium levels of skin tone and facial features are perceived as more attractive than faces with extreme levels of these factors. Black phenotypes (combinations of dark skin tone and Afrocentric facial features) were rated as more attractive than White phenotypes (combinations of light skin tone and Eurocentric facial features); ambiguous faces (combinations of Afrocentric and Eurocentric physiognomy) with medium levels of skin tone were rated as the most attractive in Experiment 2. Perceptions of attractiveness were relatively independent of racial categorization in both experiments.
\end{abstract}

Keywords: attractiveness, race, facial features, skin tone, racial categorization, mixed race 
Attractiveness as a Function of Skin Tone and Facial Features: Evidence from Categorization

\section{Studies}

Facial attractiveness affects people's lives in a wide range of social domains from mating (Rhodes, 2006; Rhodes, Simmons, \& Peters, 2005) to health (Weeden \& Sabini, 2005). A marked growth in multiracial groups in the United States-from 6.8 to 9 million people from 2000 to 2010 (US Census, 2012) — has prompted an increased attention on perceived attractiveness of faces of mixed-race individuals. Findings from cognitive and social psychology on judgments of facial attractiveness for mixed-race versus singe-race faces are largely inconclusive (e.g., Burke, Nolan, Hayward, Russell, \& Sulikowski; Lewis, 2013; Rhodes et al., 2001; Rhodes et al., 2005). Previous studies, however, have not explicitly taken into account that many facial physical characteristics determine attractiveness (e.g., skin tone, hair texture, nose shape) and systematically covary with racial/ethnic categorization. Accordingly, the main goals of the current work were to (a) assess the relative attractiveness of Black, White, and mixed-race male phenotypes, (b) investigate the relative contribution of skin tone and facial features to attractiveness judgments, and (c) examine how ratings of attractiveness map onto racial categorization judgments.

\section{Race and Attractiveness}

Research on attractiveness effects has revealed that, in accordance with the "what is beautiful is good" stereotype, individuals attribute more favorable personality traits to attractive than unattractive individuals (for review, see Eagly, Ashmore, Makhijani, \& Longo, 1991; Jackson, Hunter, \& Hudge, 1995). Attractiveness has positive consequences for many life outcomes, including social interactions (for review, see Langlois et al., 2000), health (for review, see Weeden \& Sabini, 2005), and employment and income-related outcomes (e.g., Frieze, Olson, 
\& Russell, 1991; for review, see Hosoda, Stone-Romero, \& Coat, 2003). Attractiveness also increases the chances of mating success (Rhodes, 2006; Rhodes et al., 2005) and is sought out in romantic partners (Eastwick, Luchies, Finkel, \& Hunt, 2014).

Researchers exploring which faces (e.g., single-race such as Black or White or mixedrace faces) are perceived as most attractive ${ }^{1}$ have produced somewhat inconsistent findings. For example, Burke et al. (2013) reported that both female and male Australian participants rated opposite-sex European (White) faces as more attractive than opposite-sex African (Black) faces. Interestingly, the authors also noted that women, but not men, rated African faces as more attractive than more familiar and even own-race Asian faces. Lewis (2011) found that Black male faces were perceived as more attractive than White male faces when rated by White British women. Similar findings were reported by Lewis (2012), whereby Black men were perceived as the most attractive (in comparison to Asian and White men) by British women of different racial groups (e.g., Black, White and Asian). Burke et al. (2013) argue that perceptions of masculinity and femininity drive assessment of attractiveness, with Black men perceived as more, or at least as, masculine as men from other racial groups.

A preference for mixed-race faces also exists. For example, Lewis (2011) found that women (but not men) rated mixed-race faces as more attractive than Black faces. Mixed-race faces were rated as the most attractive in comparison to faces of one's own or other races by both Japanese and Caucasian Australians (Rhodes et al., 2005). There are several explanations for mixed-race faces being perceived as the most attractive. The "averageness" hypothesis (Grammer \& Thornhill, 1994; Langlois \& Roggman, 1990) postulates that an "average face"-a composite of all faces — is preferred to any specific face and is based upon humans' general preference for prototypical exemplars (Fink \& Penton-Voak, 2002). Another is based on 
heterosis (Lewis, 2010, 2011): offspring of parents of different genetic backgrounds are considered genetically fitter, and thus, more attractive, than those of parents of similar genetic backgrounds. Yet, mixed-race faces are not always perceived as more attractive than single-race faces. In a study by Rhodes et al. (2001) Caucasian, Chinese, and mixed composite faces were rated as equally attractive by Chinese participants. Analogously, Burke et al. (2013) reported that mixed-race faces were no more attractive than single-race faces when rated by both female and male Australian participants. Therefore, our first major goal was to determine whether singlerace faces (i.e., Black and White) or mixed-race male faces are perceived as more attractive, using a more sensitive manipulation than in past research (detailed later).

\section{Skin Tone, Facial Features, and Attractiveness}

Some researchers have isolated the effects of skin tone to elucidate why Black men are sometimes perceived as more attractive. Men tend to have a darker complexion than women do (van den Berghe \& Frost, 1986). The sexual dimorphism hypothesis suggests that women with light skin tone are perceived as more attractive than women with dark skin tone, whereas the opposite is true for men (Lewis, 2011). Because Black men, on average, have a darker skin tone than White men do, they are perceived as more attractive.

Several studies indicate that light skin tone is associated with attractiveness for both male and female targets (Hill, 2002), and is preferred even by people with dark skin (Breland, 1998; Hall, 1995, 1998). Yet Wade (1996) reported higher self-attractiveness ratings in darker-skinned Black American men compared to their lighter-skinned counterparts. On the other hand, Wade and Bielitz (2005) did not find any relationship between attractiveness and skin-tone ratings of Black American men and women by White American individuals. This inconsistent evidence implies that relations between skin tone and attractiveness vary as a function of sex and race of 
the raters, self-ratings versus other-ratings, the role of context (Harvey, LaBeach, Pridgen, \& Gocial, 2005), and potentially other factors (e.g., perception of dark skin as a suntanned skin in White targets).

However, skin tone is not the only factor that predicts race classification; other facial characteristics (e.g., width of the nose, fullness of the lips) are important and could contribute to perceptions of attractiveness as well. The independent effects of facial features have not been addressed much in the literature. Notable exceptions are Wade, Irvine, and Cooper (2004) and Little, Hockings, Apicella, and Sousa (2012). Wade et al. manipulated facial racial physiognomy, creating a continuum of male faces varying from very Afrocentric to very Eurocentric, and discovered that faces with more Eurocentric features were perceived as more attractive by both White and Black American women. Little et al. manipulated facial physiognomy of female White faces (creating two versions of the same face: more and less Afrocentric) and Black faces (creating two versions of the same face: more and less Eurocentric). They reported that the White face with more Afrocentric physiognomy and the Black face with more Eurocentric physiognomy (i.e., mixed physiognomy faces) were rated as the most attractive. However, neither Wade et al. nor Little et al. manipulated skin tone.

Therefore, the second goal of our research was to investigate the relative contributions of skin tone and facial features on perceptions of attractiveness of male faces by both men and women. By investigating independent and interactive effects of the two factors, we aim to elucidate mechanisms driving differential perceptions of attractiveness in various phenotypes.

\section{Skin Tone, Facial Features, and Racial Categorization}

Recent work has investigated the contributions of both skin tone and facial features to racial categorization perceptions (e.g., Stepanova \& Strube, 2009). These researchers created 
computer-generated images using two levels of skin tone (dark vs. light) and three levels of facial features (high Afrocentric vs. low Afrocentric vs. Eurocentric). Participants rated faces with dark skin tone as more African American than those with light skin tone. Additionally, faces with highly Afrocentric facial features received highest African American ratings followed by faces with less pronounced Afrocentric facial features and then with Eurocentric facial features. The authors concluded that the effects of skin tone and facial features are independent and additive. However, subsequent studies revealed that their influence is more complex. When a more sensitive manipulation of skin tone and facial features was employed (10 levels of skin tone and 10 levels of facial features), an interaction between skin tone and facial features on racial typicality judgments was noted (Dunham et al., 2015; Stepanova \& Strube, 2012a; Stepanova et al., 2013). Although skin tone was always an important marker for racial categorization judgments, the impact of facial features became more prominent as skin tone became lighter. Accordingly, our third major goal was to use these more sensitive methods to determine the relative contribution of skin tone and facial physiognomy to attractiveness judgments and to determine how attractiveness and racial categorization judgments are related.

\section{Overview of Studies}

We assessed perceptions of attractiveness in multiple racial phenotypes (e.g., White, Black and mixed-race) and explored interactive and additive effects of skin tone and facial features on such perceptions (Experiment 1 and 2). For Experiment 1, we used a set of six faces, varying in facial features, (3 levels) and skin tone (2 levels). In Experiment 2, we used a more sensitive manipulation of skin tone and facial features (10 levels of skin tone, ranging from dark to light, crossed with 10 levels of facial features, ranging from very Afrocentric to very 
Eurocentric). In both experiments, we compared perceptions of racial typicality and perceptions of attractiveness.

We predicted that mixed-race faces would be rated as the most attractive in both Experiments 1 and 2, followed by the Black and White faces. We were not certain whether Black faces would be perceived as more attractive than White faces, given mixed evidence from previous studies. We do not offer specific predictions about either facial features main effects or Skin tone $\mathrm{x}$ Facial Features interactions for Experiments 1 and 2. There is limited previous work on contributions of facial features to perceptions of attractiveness, and especially so because our work is the first, to our knowledge, to employ fine orthogonal manipulations of skin tone and facial physiognomy in perceptions of attractiveness. Analogously, our comparison of the attractiveness and racial categorization judgments was largely exploratory (Experiment 1 and 2).

\section{Experiment 1}

The goals of Experiment 1 were to identify the influence of skin tone and facial features on attractiveness ratings, and to determine the relationship between perceptions of attractiveness and racial typicality/racial categorization. ${ }^{2}$

\section{Method}

\section{Participants}

Participants for this and all subsequent experiments were recruited from a mid-size private university located in the Midwestern United States. The study sample comprised of 85 undergraduate students ( 81 women and 4 men, $M$ age $=19.2, S D=1.11$ ). The sample comprised 13 Asians/Asian Americans, 6 Blacks/African Americans, 60 Whites/European Americans, 3 Latino/Hispanic Americans, 2 Mixed, and 1 Other.

\section{Stimuli and Procedure}


Facial features were manipulated using Poser $6^{\mathrm{TM}}$ software to produce a high Afrocentric physiognomy (HP) face, a low Afrocentric physiognomy (LP) face, and a Eurocentric physiognomy (E) face. Software controls allowed simultaneous manipulation of feature sets (nose, lips, face and head shape), we also altered some individual features. Two skin tone versions of each face (light and dark) were also created. Half of the participants saw faces in monochromatic (gray-scale) mode and half of the participants saw faces in polychromatic (color) mode. Finally, some participants completed the racial typicality/categorization judgments first, and some completed the attractiveness judgment first. Thus, we employed a 3 (Facial Physiognomy) x 2 (Skin Tone) x 2 (Color presentation mode) x 2 (Task Order) mixed design with the last two factors manipulated between participants.

Participants completed all phases of the experiment (a typicality rating task, an attractiveness rating task, a racial categorization task, and a demographics questionnaire) via computer in under 30 minutes. The typicality judgment was made on a 7-point scale: 1 (very African American), 2 (moderately African American), 3 (somewhat African American), 4 (not clearly African American or European American), 5 (somewhat European American), 6 (moderately European American), and 7 (very European American). Analogously, for the attractiveness judgment task, participants used a 7-point scale, ranging from 1 (very unattractive) to 7 (very attractive). For the racial categorization task, participants used a 3category judgment: 1 (African American), 2 (cannot tell), and 3 (European American). The presentation order of faces was randomized separately for each task and participant; in each of the three tasks, each face was presented only once (i.e., 6 trials per task, 18 trials total). After the first three tasks, participants completed a demographic questionnaire, were debriefed and then dismissed. 


\section{Results and Discussion}

Attractiveness ratings. First, we performed an analysis of variance of the attractiveness ratings using two within-subjects variables (facial physiognomy and skin tone) and two betweensubjects (mode of presentation and task order) variables. Bonferroni corrections were applied for follow-up comparisons. Only significant findings are reported below. For attractiveness ratings, there were significant main effects for skin tone, $F(1,81)=76.42, p<.001, \eta_{\mathrm{p}}{ }^{2}=.49$; physiognomy, $F(2,80)=136.40, p<.001, \eta_{\mathrm{p}}{ }^{2}=.63$, and mode of presentation, $F(1,81)=5.45, p$ $=.02, \eta_{\mathrm{p}}{ }^{2}=.063$. Darker faces $(M=4.64, S E=0.10)$ were perceived as more attractive than lighter faces $(M=3.86, S E=0.98)$. Faces with a greater degree of Eurocentric physiognomy were perceived as more attractive than faces characterized by a greater degree of Afrocentric physiognomy: E $(M=5.15, S E=0.12), \mathrm{LP}(M=4.54, S E=0.11)$, and HP $(M=3.05, S E=0.12)$. When faces were presented in the monochromatic mode $(M=4.45, S E=0.12)$, they were rated as more attractive than when presented in the polychromatic mode $(M=4.04, S E=0.13)$. As Figure 1 illustrates, skin tone and physiognomy main effects were qualified by a significant Physiognomy $\times$ Skin Tone interaction, $F(2,81)=6.20, p=.003, \eta_{\mathrm{p}}{ }^{2}=.071$, indicating that skin tone differences were most pronounced for HP Afrocentric faces (1.05 mean difference between dark and light faces) than LP Afrocentric (.81 mean difference) and Eurocentric faces (.49 mean difference) (all $p \mathrm{~s}<.05)$. This interaction accounts for far less variance than the physiognomy and skin tone main effects.

Because mode of presentation and task order did not qualify the skin tone and physiognomy findings, we conducted all the subsequent analyses on attractiveness data without these factors. Because some of the racial/ethnic groups were represented by very low frequencies, we recoded ethnicity into White and non-White and tested this additional factor in 
the analysis of attractiveness judgments. No significant effects or interactions involving this factor emerged (all $p \mathrm{~s}>.70$ ). We did not test sex of participants as a factor in any of the analyses because only four participants were male and removal of their data did not alter the pattern of results.

Racial typicality ratings. For typicality ratings, we collapsed our data across Color Presentation Mode and Task Order factors, since racial typicality ratings are not the main focus of this work. Racial typicality and categorization ratings data were partially reported (for White female participants only, $N=59$ ) in Stepanova and Strube (2009). They are summarized here for the full sample briefly to provide a comparison with attractiveness ratings.

There were significant main effects for skin tone $\left[F(1,81)=82.82, p<.001, \eta_{\mathrm{p}}{ }^{2}=.50\right]$ and physiognomy $\left[F(2,80)=415.68, p<.001, \eta_{\mathrm{p}}{ }^{2}=.83\right]$. Darker faces $(M=2.92, S E=.08)$ were perceived as more African American than lighter faces $(M=3.98, S E=.08)$. High Afrocentric physiognomy faces were perceived as the most African American/least European American $(M=1.81, S E=.07)$, followed by low Afrocentric physiognomy faces $(M=3.19, S E=$ $.10)$, and then by Eurocentric faces $(M=5.35, S E=.10)$. As Figure 2 illustrates, a significant Physiognomy x Skin Tone interaction emerged, $F(2,168)=3.16, p=.045, \eta_{\mathrm{p}}{ }^{2}=.036$. Skin tone differences between dark and light faces were the most pronounced for Eurocentric faces (1.28 mean difference) than LP Afrocentric (.93 mean difference) or HP Afrocentric faces (.94 mean difference) (all $p s<.05)^{3}$. Light skin tone (in comparison to dark) increases perception of faces as being more European American/less African American, and especially so for Eurocentric faces. When ethnicity was entered in the analysis, no significant effects or interactions involving this factor emerged (all $p \mathrm{~s}>.70$ ). 
The correlation between attractiveness ratings and typicality ratings within each stimulus condition (average $|r|=.12$ ) suggests that participants were attending to different features when making racial typicality versus attractiveness judgments.

Our results indicate that both dark skin tone and African facial physiognomy influence attractiveness ratings, but in opposite directions. The most important novel contribution of this work is that these two factors interact: perceived attractiveness of faces, but especially HP Afrocentric faces, was enhanced by the dark skin tone. The most attractive faces (out of 6) were a dark Eurocentric face and a dark face characterized by low physiognomy Afrocentric features. Such faces were perceived as mixed-race faces; the racial typicality ratings of 4.71 and 3.65 ratings, respectively, were closest to the midpoint on the racial typicality scale. Hence, these findings are consistent with our hypothesis and some of the previous work indicating that mixedrace faces are perceived as more attractive than either Black faces (Lewis, 2011) or single-race faces of different races (Rhodes et al., 2005). Yet, White faces of Eurocentric physiognomy with light skin tone were also rated as highly attractive by our study participants, in line with some of the previous research reviewed (Burke et al., 2013).

Although Experiment 1 provides initial important evidence of (a) differences in ratings of mixed-race and single-race faces, (b) interactive effects of skin tone and facial features and (c) independence of racial categorization versus attractiveness judgments, it was based on a predominately White female sample and used a limited number of skin tone and facial features levels. Experiment 2 aimed to address these limitation.

\section{Experiment 2}

In Experiment 2, we used a stimulus set (same as Dunham et al., 2015; Stepanova \& Strube, 2012a; Stepanova et al., 2013) with finer gradations of skin tone and facial physiognomy: 
10 levels of skin tone (from dark to light) and 10 levels of facial physiognomy (from very Afrocentric to very Eurocentric). The goals of the experiment were to (a) assess perceptions of attractiveness in multiple racial phenotypes beyond those used in Experiment 1, (b) determine specific contributions of skin tone and facial features on perceptions of attractiveness using a finer manipulation of both factors, and (c) further explore the relationship between perceptions of attractiveness and racial typicality.

\section{Method}

\section{Participants}

Participants were 321 undergraduate students $\left(M_{\mathrm{age}}=19.20, S D=1.36,56.1 \%\right.$ female $)$, comprising 64.8\% Whites/European Americans, 16.5\% Asian/Asian Americans, 6.2\% Black/African Americans, 2.8\% Latino/Hispanic Americans, and 9.7\% Mixed or Other.

\section{Stimuli and Procedure}

We created six facial stimuli sets (grids), each developed from a unique pair of one Black (dark Afrocentric) face and one White (light Eurocentric) face using Poser $6^{\mathrm{TM}}$ software. A procedure employed for the creation of a facial grid was similar to Experiment 1.

Each grid varied with respect to skin tone (gradually changing from dark to light, across ten levels) and in terms of degree of Afrocentric and Eurocentric facial physiognomy (ranging from very Afrocentric to very Eurocentric), see Figure 3 for a sample set. Thus, the experiment employed a 10 (Skin Tone) x 10 (Facial Physiognomy) within-subjects design.

Participants completed the computer-administered tasks individually in under 1.5 hours. The set presented to a participant was randomly selected from the available six. Participants were asked to rate faces on attractiveness, racial typicality, and several emotional expressions (anger, happiness, and sadness). The order of the rating tasks was randomly determined. For 
each of the five tasks, participants rated all 100 faces (500 trials total); they were presented with one face at a time, and were given unlimited time for its evaluation. Once they provided a rating for the face, a new face was automatically shown. Order of the faces within task was randomized separately for each participant. For the attractiveness task, participants were asked to rate the face on a scale ranging from 1 (Not at all attractive) to 9 (Very attractive). For the racial typicality task, participants were asked to rate the face on a scale ranging from 1 (Very African American) to 9 (Very European American). For the emotional expression rating tasks, participants were asked to rate the face on a scale ranging from 1 (Not at all angry/happy/sad) to 9 (Very angry/happy/sad).

Additionally, participants completed two rank-ordering tasks in order to determine if the faces could be reliably distinguished along the manipulated dimensions. They were instructed to rank-order faces based on (a) skin tone and (b) facial physiognomy. In each of these two tasks, they were presented with either one row of faces (for skin judgments) or one column of faces (for facial physiognomy judgments) from the $10 \times 10$ grid of faces. The column or row a participant saw in each task was randomly determined. These tasks were included to determine whether the gradations in skin tone and facial physiognomy are perceived as intended.

Participants were either presented with the rank-order tasks first or the rating tasks first. At the end of the study, participants were asked to indicate their age, gender, and ethnicity before being debriefed and dismissed.

\section{Results and Discussion}

We used the Greenhouse-Geisser corrected degrees of freedom for the $F$ tests whenever the assumption of sphericity was violated, as indicated by Mauchly's test of sphericity. This statistical procedure reduces the degrees of freedom (in proportion to the violation), resulting in 
values that are not whole numbers. We conducted all follow-up comparisons with Bonferroni corrections at the level of the effects under examination.

Attractiveness ratings. The 10 (Skin Tone) $\times 10$ (Facial Physiognomy) repeated measures multiple regression revealed significant main effects for both factors. Faces that were characterized by medium skin tone were rated as more attractive than were faces with either darker or lighter skin tone, $F(3.26,1043.79)=69.94, p<.001, \eta_{\mathrm{p}}{ }^{2}=.18$. Moreover, faces that were medium in physiognomy (mixed Eurocentric and Afrocentric features) were rated as more attractive than faces with more Afrocentric or Eurocentric physiognomy, $F(1.81,577.66)=$ $72.81, p<.001, \eta_{\mathrm{p}}{ }^{2}=.19$. Within each of these factors, for the majority of pairwise comparisons, each mean was significantly different from every other mean $(p<.05$, Bonferroni adjustment) with several exceptions. The means, standard errors and results of the pairwise comparisons are presented in Table 1.

We also found a significant Skin Tone $\times$ Facial Physiognomy interaction, which is shown in Figure 4, $F(50.06,16018.82)=26.59, M S E=45.21, p<.001, \eta_{\mathrm{p}}{ }^{2}=.08$. Overall, faces that received the most attractive ratings were characterized by medium physiognomy levels, i.e., within skin levels 4-8 (medium). More specifically, the Skin Tone $\times$ Facial Physiognomy interaction indicated significant linear $\left.\left[F(1,320)=2947.93, p<.001, \eta_{\mathrm{p}}{ }^{2}=.55\right)\right]$, quadratic $[F(1$, $\left.\left.320)=6.09, p=.01, \eta_{\mathrm{p}}{ }^{2}=.02\right)\right]$, and cubic $\left.\left[F(1,320)=137.70, p<.001, \eta_{\mathrm{p}}{ }^{2}=.30\right)\right]$ trends. Faces with strong Afrocentric physiognomy (Facial 1-3) received the highest attractiveness ratings when skin tone was dark with attractiveness ratings declining as skin tone became lighter. Faces with evenly mixed Afrocentric and Eurocentric features (see levels 5 and 6) received the lowest ratings when the skin tone was either dark or light, steadily rising and peaking at the midlevels of skin tone (levels 5-6). Faces with strong Eurocentric physiognomy (Facial 8-10) 
received the lowest attractiveness ratings when skin tone was dark, with attractiveness ratings steadily increasing until skin levels reached level 7-8 (lighter skin) and declining for the lightest skin tone. When sex of the participants was entered in the analyses, there was a significant Facial Physiognomy $\times$ Sex interaction, which is shown in Figure $5, F(1.80,574.81)=6.83, p=.002$, $\eta_{\mathrm{p}}{ }^{2}=.02$, Women rated faces with mixed Afrocentric and Eurocentric physiognomy (levels 4-6) as more attractive than men did (all $p<.05$, Bonferroni adjustment); however, men rated faces with the most prominent Eurocentric physiognomy as more attractive than women did.

Racial typicality ratings. Results of racial typicality ratings were analogous to those reported previously in other papers using similar 10 x 10 face grids employed in this study (e.g., Dunham et al., 2015; Stepanova \& Strube, 2012a; Stepanova et al., 2013) and were mainly measured to assess their relationship to attractiveness ratings, thus, we describe them here briefly. Darker faces were rated as more African American than lighter faces, $F(1.76,563.72)=$ 230.46, $p<.001, \eta_{\mathrm{p}}{ }^{2}=.45$, and Afrocentric faces were rated as more African American than Eurocentric faces, $F(1.58,506.12)=871.76, p<.001, \eta_{\mathrm{p}}{ }^{2}=.73$. A significant Skin tone $\times$ Facial Physiognomy interaction $\left[F(54.22,17349.54)=9.22, M S E=20.94, p<.001, \eta_{\mathrm{p}}{ }^{2}=.03\right]$ indicated that when skin tone was dark, participants' ratings were less variable; however, as the skin tone became lighter, racial typicality ratings became more variable and depended more on facial physiognomy. Ethnicity/race of the participants did not moderate any of the racial typicality or attractiveness effects described (it was entered in analyses as a binary factor analogous to Experiment 1).

We computed a correlation between the profiles of attractiveness means and typicality means, with the means calculated for each face (profiles of 100 means in each case), $r(1)=.10, p$ 
$=.30$. This suggests that participants pay attention to different cues when judging racial typicality versus attractiveness.

Manipulation check. The manipulation of skin tone and facial features was successful. A Friedman test indicated that faces were ranked differently on the basis of their facial features, $\chi^{2}(9)=2064.90, p<.001$ and skin tone, $\chi^{2}(9)=2019.09, p<.001$. Mean ranks for both factors are reported in Table 2. Each level of skin tone was ranked differently from adjacent ones (all $p \mathrm{~s}$ $<.001$ ), and each level of facial physiognomy was ranked differently from adjacent ones (all $p \mathrm{~s}$ $<.001)$, indicating that incremental differences in both skin tone and facial physiognomy are perceived by participants as such.

Our results ${ }^{4}$ indicate that mixed Afrocentric-Eurocentric faces with medium levels of skin tone (mixed-race) were deemed the most attractive, followed by dark Afrocentric (Black) faces and then by light Eurocentric (White) faces. These findings support the "averageness" hypothesis (Grammer \& Thornhill, 1994; Langlois \& Roggman, 1990) because faces exhibiting Black phenotypes (dark skin and Afrocentric physiognomy) and White phenotypes (light skin tone and Eurocentric physiognomy) were rated as less attractive than "average" faces (of mixed physiognomy and mixed skin tone) by both female and male participants. Our findings suggest that disparities in perception of facial attractiveness go above and beyond skin tone, and are in fact equally driven by complexities of facial features (as indicated by the effect sizes as well). Moreover, the dark(est) skin tone is not perceived as the most attractive skin tone; rather, skin tones in the middle of the darkness scale are perceived as the most attractive. Therefore, we do not believe that differences in perceptions of attractiveness can be easily explained by sexual dimorphism in skin tone. 
An evolutionary approach would suggest that these effects would be moderated by the gender of perceivers. This was not the case in our study; gender did not moderate the skin tone effects. On the other hand, gender did moderate the influence of facial features. In accordance with the evolutionary heterosis approach, women rated faces exhibiting "average" physiognomy as more attractive than men did.

The complex pattern of the Skin Tone $\times$ Facial Physiognomy interaction, however, suggests that perceptions of attractiveness as a function of skin tone vary across physiognomy levels. Interestingly, perceived attractiveness of Afrocentric faces was enhanced by the dark skin tone, while medium or light skin tone had the opposite effect. For Eurocentric faces, perception of attractiveness was decreased by dark skin tone, and enhanced by lighter skin tones, although not by extremely light skin tone (9-10). Notably, medium skin tone does not enhance attractiveness ratings of Afrocentric faces, but it does increase attractiveness of mixed and Eurocentric faces. These findings indicate that race-based attractiveness judgments are highly sensitive to variations in multiple markers. Importantly, consistent with the findings of Experiment 1, attractiveness and racial typicality judgments were not strongly related.

\section{General Discussion}

\section{Race and Attractiveness}

Experiment 1 provided initial evidence that faces exhibiting mixed features (dark Eurocentric and dark mixed physiognomy face) are perceived as the most attractive; Experiment 2 findings confirmed this pattern, as mixed-race faces were rated as the most attractive, followed by Black faces, with White faces perceived as the least attractive.

Notably, in Experiment 1, Black faces were not rated as more attractive than White faces, while Black faces were perceived as more attractive than White faces in Experiment 2. Faces 
representing White phenotype (see the lower right corner, Figure 3) received a racial typicality rating corresponding to a $z$-score of 5.4 (close to the very European American end-point of the scale) in Experiment 2. Faces representing Black phenotype (see the upper left corner, Figure 3) received a racial typicality $z$ score of -4.80 (close to the very African American end-point of the scale) in Experiment 2. Yet Experiment 1 racial typicality z-scores for White phenotype (the last face on the right, see Figure 2) and Black phenotype (the first face on the left, see Figure 2) are clearly less extreme (4.4 and -3.64 correspondingly). We also computed average racial typicality $z$ scores for (a) 3 faces representing White phenotype and (b) 3 faces representing Black phenotype in Experiment 2 (3 faces composing the lower right corner and 3 faces composing the upper left corner, see Figure 3). These scores were 5.17 (White) and -.4.75 (Black) correspondingly, which are more extreme than z-scores for White and Black phenotypes in Experiment 1. We are inclined to believe that end-points (White and Black phenotypes) in Experiment 2 represent their respective racial categories better than end-points in Experiment 1. Results from Experiment 2 are also more generalizable because they do not rely on idiosyncratic properties of stimuli, since they are based on more than one set of faces (six unique grids of faces were used in Experiment 2 and only one was used in Experiment 1). Therefore, we conclude that Black male phenotypes are perceived as more attractive than White phenotypes based on findings from Experiment 2.

Collectively, these studies clearly show that mixed-race faces are perceived as the most attractive; and that Black faces are perceived as more attractive than White faces, providing additional evidence to support the assertion that Black male faces are universally perceived as more attractive than White faces. Perhaps these findings can explain some of the disparities in interracial marriages among men. The most recent statistics on interracial marriage suggests that 
Black men are more likely to enter an interracial marriage than their White or Asian counterparts (Pew Research Center, 2015). Among other reasons, their perceived attractiveness might be one of the factors driving this trend.

\section{Facial Features, Skin Tone, and Attractiveness}

Some authors (Lewis, 2011) argue that dark skin tone is the main contributor to high attractiveness ratings in Black males, citing the concept of sexual dimorphism. Our work allowed us to disambiguate the role of skin tone. Skin tone, although a powerful marker of attractiveness, did not simply increase attractiveness as it becomes darker. In fact, faces that were of medium skin tone were perceived as the most attractive, challenging the widely held assumption that dark skin tone in men is the most attractive. Race-based attractiveness cannot be explained by reliance on skin tone alone.

At the same time, we would like to caution readers against juxtaposition of our results with those yielded by previous studies. The operationalization of skin tone in other studies likely differed from our own; it is possible that dark skin tone used in prior research is substantially lighter than the darkest skin tone we employed in our work. Even in our own work, extreme levels of skin tone (e.g. very dark and very light) were operationalized differently in Experiments 1 and 2 (as illustrated by Figures 1 and 2). For example, end-points for "darkest Eurocentric face" are not identical across these two studies and, thus, differences in their ratings can be explained by different operationalization of two manipulated factors. Additional differences such as variability in samples and nature of rating tasks (e.g., 6 versus 100 trials per task) also make it difficult to compare results across studies.

Most importantly, we have shown that both skin tone and facial physiognomy markers contribute interactively to perceptions of attractiveness. The contribution of skin tone varied 
across physiognomy levels, clarifying which mixed phenotypes are perceived as the most attractive. Notably, these faces are "true" mixtures with almost equal Afrocentric and Eurocentric physiognomy and of medium skin tone (supporting the "averageness" hypothesis put forth by Langlois \& Roggman, 1990; see also Grammer \& Thornhill, 1994). Attractiveness ratings of mixed physiognomy faces were described by a quadratic function. Such faces received the lowest ratings when the skin tone was either dark or light, with ratings steadily rising and peaking at the mid-levels of skin tone. Attractiveness of faces of Afrocentric or Eurocentric physiognomy followed a different pattern. Faces with Afrocentric physiognomy received the highest attractiveness ratings when skin tone was dark, which declined as skin tone became lighter. Faces with Eurocentric physiognomy received the lowest attractiveness ratings when skin tone was dark, with attractiveness ratings steadily increasing until skin levels reached lighter skin levels and declining for the lightest skin tone. The described effect for Afrocentric faces was confirmed in both Experiment 1 and 2. These results indicate a potential "expectancy violation" effect (Burgoon \& Jones, 1976), whereby extreme mismatches between skin tone and facial physiognomy (dark skin tone and Eurocentric physiognomy OR light skin tone and Afrocentric physiognomy) produce the lowest attractiveness ratings. Dark skin tone enhances attractiveness of Afrocentric faces, resulting in Black phenotypes, whereas medium skin tone increases attractiveness of mixed and Eurocentric faces. These results challenge previous assumptions about contributions of dark skin tone to perceptions of attractiveness in men (e.g., Breland, 1998; Hall, 1995, 1998; Hill, 2002), portraying a more complex picture.

\section{Gender and Race Effects of Perceivers}

Importantly, the effects described here were not moderated by the gender or ethnicity of the perceivers, even though the majority of our participants were women. Most importantly, 
male faces with mixed physiognomy and mixed skin tone were perceived as the most attractive by individuals irrespective of the perceiver's gender or race. Only one effect in Experiment 2 was qualified by the gender of participants, whereby women rated faces of "average" physiognomy as more attractive than men did. Although findings that male faces with mixed physiognomy and mixed skin tone are perceived as the most attractive can be explained by either humans' general preference for prototypical examples or the heterosis, women's higher ratings of mixed physiognomy faces would indicate that the evolutionary explanation still has merit.

\section{Racial Typicality Judgments and Attractiveness Judgments are Independent}

In both Experiment 1 and 2, racial typicality and attractiveness judgments were not highly correlated. People appear to attend to different features/weigh them differently when making these judgments; we believe that this work has practical implications for researchers who use facial stimuli of different races: matching stimuli of different races on attractiveness might produce divergent results for perceived racial typicality.

\section{Limitations and Future Directions}

For both studies discussed here, we only employed computer generated male faces, predominately because we wished to build upon previous work with these sets of stimuli (i.e., Chen, Couto, Sacco, \& Dunham, in press; Dunham et al., 2015; Stepanova \& Strube, 2012a, 2012b; Stepanova et al., 2013). An important task for future research will be extending the methods to female stimuli.

The majority of the participants in Experiment 1 and 2 were women. Importantly, when participants of both genders were included, no gender effects were recorded, except for the Facial Physiognomy by Gender interaction in Experiment 2. Analogously, although participants of different ethnicities took part in Experiment 1 and 2, there were no effects of participants' 
ethnicity (when limited to a White vs. non-White comparison). The absence of these effects suggests generalizability of the explicit judgments of attractiveness, at least in the Northern American/Western cultural context. Nonetheless, there were relatively few participants in the several non-White groups, limiting the conclusions that can be drawn about ethnic/racial moderators. This remains an important task for future research. Including facial matrixes of other racial physiognomies such as Asiancentric/Eurocentric physiognomy (see Dunham et al., 2016) or Asiancentric/Afrocentric physiognomy, participants of other racial groups and utilizing female stimuli could produce results with broader implications.

Finally, a potential concern can be raised whether the measures of attractiveness we used tap into an entirely different construct: racial prejudice. Social psychological research has long argued that attractiveness is related to overall liking/favorability of evaluations (e.g., Byrne, London, \& Reeves, 1968; Miller, 1970), but we have not measured racial bias in our samples, so we cannot answer this question empirically. Future research should address this. Yet we also would like to remind our readers that measures of explicit attractiveness we used are common in attractiveness research.

\section{Conclusion}

This work indicates that faces of mixed racial phenotypes (when skin tone is mediumdark and facial features are an even mixture of Afrocentric and Eurocentric features) are perceived as the most attractive, followed by Black phenotypes, with White phenotypes perceived as least attractive. There is a complex relationship between skin tone and facial physiognomy determining attractiveness ratings. Perceptions of attractiveness might not be driven by racial categories, but by multiple facial characteristics. Importantly, individuals attend to different features when making explicit racial typicality versus attractiveness judgments. 


\section{References}

Breland, A. M. (1998). A model for differential perceptions of competence based on skin tone among African-Americans. Journal of Multicultural Counseling and Development, 26 (4), 294-312. doi:10.1002/j.2161-1912.1998.tb00206.x

Burgoon, J. K., \& Jones, S. B. (1976). Toward a theory of personal space expectations and their violations. Human Communication Research, 2, 131-146. doi:10.1111/j.14682958.1976.tb00706.x

Burke, D., Nolan, C., Hayward, W. G., Russell, R., \& Sulikowski, D. (2013). Is there an ownrace preference in attractiveness? Evolutionary Psychology, 11(4), 855-872. doi: $10.1177 / 147470491301100410$

Byrne, D., London, O., \& Reeves, K. (1968). The effects of physical attractiveness, sex, and attitude similarity on interpersonal attraction. Journal of Personality, 36, 259-271. doi:10.1111/j.1467-6494.1968.tb01473.x


(Black or Multiracial or White): Cultural variation in racial boundaries. Social Psychological and Personality Science.

Dunham, Y. Dotsch, R., Clark A. R., \& Stepanova, E. V. (2016). The development of White-Asian categorization: Contributions from skin color and other physiognomic cues. PLOS ONE, 11(6): e0158211. doi:10.1371/journal.pone.0158211

Dunham, Y., Stepanova, E. V., Dotsch, R., \& Todorov, A. (2015). The development of racebased perceptual categorization: skin color dominates early category judgments. Developmental Science, 18, 469-483. doi: 10.1111/desc.12228.

Eagly, A. H., Ashmore, R. D., Makhijani, M. G., \& Longo, L. C. (1991). What is beautiful is 
good, but...: A meta-analytic review of research on the physical attractiveness stereotype. Psychological Bulletin, 110(1), 109-128. doi:10.1037/0033-2909.110.1.109

Eastwick, P. W., Luchies, L. B., Finkel, E. J., \& Hunt, L. L. (2014). The predictive validity of ideal partner preferences: A review and meta-analysis. Psychological Bulletin, 140(3), 623-665. doi:10.1037/a0032432

Fink, B., \& Penton-Voak, I. (2002). Evolutionary psychology of facial attractiveness. Current Directions in Psychological Science,11(5), 154-158. doi:10.1111/1467-8721.00190

Frieze, I. H., Olson, J. E., \& Russell, J. (1991). Attractiveness and income for men and women in management. Journal of Applied Social Psychology, 21(13), 1039-1057. doi:10.1111/j.1559-1816.1991.tb00458.x

Grammer, K., \& Thornhill, R. (1994). Human (Homo sapiens) facial attractiveness and sexual selection: The role of symmetry and averageness. Journal of Comparative Psychology, 108(3), 233-242. doi:10.1037/0735-7036.108.3.233

Harvey, R. D., LaBeach, N., Pridgen, E.,\& Gocial, T. M. (2005). The intragroup stigmatization of skin tone among Black Americans. Journal of Black Psychology, 31, 237-253. doi:10.1177/0095798405278192

Hall, R. (1995). The bleaching syndrome: African Americans' response to cultural domination vis-à-vis skin tone. Journal of Black Studies, 26 (2), 172-184.

Hall, R. E. (1998). Skin tone bias: A new perspective on an old social problem. The Journal of Psychology, 132(2), 238-240. doi:10.1080/00223989809599163

Hill, M. E. (2002). Skin tone and the perception of attractiveness among African Americans: Does gender make a difference? Social Psychology Quarterly, 65, 77-91.

Hosoda, M., Stone-Romero, E. F., \& Coats, G. (2003). The effects of physical attractiveness on 
job-related outcomes: A meta-analysis of experimental studies. Personnel Psychology, 56(2), 431-462. doi:10.1111/j.1744-6570.2003.tb00157.x

Jackson, L. A., Hunter, J. E., \& Hodge, C. N. (1995). Physical attractiveness and intellectual competence: A meta-analytic review. Social Psychology Quarterly, 58(2), 108-122. doi:10.2307/2787149

Langlois, J., Kalakanis, L., Rubenstein, A., Larson, A., Hallam, M., \& Smoot, M. (2000). Maxims or myths of beauty? A meta-analytic and theoretical review. Psychological Bulletin, 126(3), 390-423. doi:10.1037/0033-2909.126.3.390

Langlois, J. H., \& Roggman, L. A. (1990). Attractive faces are only average. Psychological Science, 1(2), 115-121. doi:10.1111/j.1467-9280.1990.tb00079.x

Little, A. C., Hockings, K. J., Apicella, C. L., \& Sousa, C. (2012). Mixed-ethnicity face shape and attractiveness in humans. Perception, 41(12), 1486-1496. doi:10.1068/p7278

Lewis, M. B. (2010). Why are mixed-race people perceived as more attractive? Perception, 69, 136-138. doi:10.1068/p6626

Lewis, M. B. (2011). Who is the fairest of them all? Race, attractiveness and skin tone sexual dimorphism. Personality and Individual Differences, 50(2), 159-162. doi:10.1016/j.paid.2010.09.018

Lewis, M. B. (2012). A facial attractiveness account of gender asymmetries in interracial marriage. Plos ONE, 7(2), doi:10.1371/journal.pone.0031703

Miller, A. G. (1970). Role of physical attractiveness in impression formation. Psychonomic Science, 19, 241-243. doi: 10.3758/BF03328797

Pew Research Center. (2015). Multiracial in America: Proud, Diverse and Growing in Numbers. 
Retrieved from: http://www.pewresearch.org/fact-tank/2015/06/12/interracial-marriagewho-is-marrying-out/

Rhodes, G., Yoshikawa, S., Clark, A., Lee, K., McKay, R., \& Akamatsu, S. (2001).

Attractiveness of facial averageness and symmetry in non-Western cultures: In search of biologically based standards of beauty. Perception, 30(5), 611-625. doi:10.1068/p3123

Rhodes, G. (2006). The evolutionary psychology of facial beauty. Annual Review of Psychology, 57, 199-226. doi:10.1146/annurev.psych.57.102904.190208

Rhodes, G., Lee, K., Palermo, R., Weiss, M., Yoshikawa, S., Clissa, P., \& ... Jeffery, L. (2005). Attractiveness of own-race, other-race, and mixed-race faces. Perception, 34(3), 319-340. doi:10.1068/p5191

Rhodes, G., Simmons, L. W., \& Peters, M. (2005). Attractiveness and sexual behavior: Does attractiveness enhance mating success? Evolution and Human Behavior, 26(2), 186-201. doi:10.1016/j.evolhumbehav.2004.08.014

Stepanova, E. V., \& Strube, M. J. (2009). Making of a face: Role of facial physiognomy, skin tone and color presentation mode in evaluations of racial typicality. The Journal of Social Psychology. 149 (1), 66-81. doi: 10.3200/SOCP.149.1.66-81

Stepanova E. V. \& Strube, M. J (2012a). The role of skin tone and facial physiognomy in racial categorization: Moderation by implicit racial attitudes. Journal of Experimental Social Psychology. 48, 867-878. doi: 10.1016/j.jesp.2012.02.019

Stepanova, E. V., \& Strube, M. J (2012b). What's in a face? The role of skin tone, facial physiognomy, and color presentation mode of facial primes in affective priming effects. The Journal of Social Psychology. 152 (2), 212-227. doi:10.1080/00224545.2011.597797 
Stepanova, E. V., Strube, M. J, \& Yablonsky, G. S. (2013). Now one is Russian, now one is not: Ethnic attitudes and categorization effects in the Russian Federation. International Perspectives in Psychology: Research, Practice, Consultation, 2(2), 85-99. doi: $10.1037 / \mathrm{a} 0032339$

US Census (2012). The two or more races population: 2010. Retrieved from: https://www.census.gov/prod/cen2010/briefs/c2010br-13.pdf

van den Berghe, P. L. \& Frost, P. (1986). Skin tone preference, sexual dimorphism and sexual selection: A case of gene-culture co-evolution? Ethnic and Racial Studies, 9, 87-113. doi: 10.1080/01419870.1986.9993516

Wade, T. J. (1996). The relationships between skin tone and self-perceived global, physical, and sexual attractiveness, and self-esteem for African-Americans. Journal of Black Psychology, 22, 358-373. doi: 10.1177/00957984960223006

Wade, T. J., \& Bielitz, S. (2005).The differential effect of skin tone on attractiveness, personality evaluations, and perceived life success of African Americans. Journal of Black Psychology, 31 (3), 215-236. doi: 10.1177/0095798405278341

Wade, T. J., Irvine, K., \& Cooper, M. (2004). Racial characteristics and individual differences in women's evaluations of men's facial attractiveness and personality. Personality and Individual Differences, 36, 1083-1092. doi:10.1016/S0191-8869(03)00202-2

Weeden, J., \& Sabini, J. (2005). Physical Attractiveness and Health in Western Societies: A Review. Psychological Bulletin, 131(5), 635-653. doi:10.1037/0033-2909.131.5.635 


\section{Footnotes}

${ }^{1}$ When reviewing pertinent studies, we will primarily focus on those examining how faces of European/White, African/Black, and mixed descent (Euro-African) are perceived in terms of attractiveness.

${ }^{2}$ Note that partial data from Experiment 1 were published in Stepanova and Strube (2009): racial typicality and categorization ratings only for White female participants $(N=59)$.

${ }^{3}$ We acknowledge redundancy of the racial categorization task, given that it captures the same information as the racial typicality task. We originally included the racial categorization task to test if it will be able to detect all the same effects as the racial typicality task. Yet the racial categorization task appears to be less sensitive to our skin tone and facial physiognomy manipulations. A parallel analysis on the racial categorization ratings revealed the same main effects for Skin Tone $\left[F(1,84)=29.71, p<.01, \eta_{\mathrm{p}}{ }^{2}=.26\right]$ and Facial Physiognomy $[F(2,83)=$ $\left.607.03, p<.01, \eta_{\mathrm{p}}{ }^{2}=.94\right]$, though a Skin Tone $\mathrm{x}$ Facial Physiognomy interaction did not reach significance $(p=.12)$. The pattern of results for two main effects was identical to the results in the racial typicality task, with dark faces $(M=1.75, S E=.03)$ rated as more African American than light faces $(M=2.06, S E=.04)$. HP faces were perceived as the most African American/least European American $(M=1.11, S E=.03)$, followed by LP faces $(M=1.80, S E=$ $.06)$, and then by Eurocentric faces $(M=2.81, S E=.03)$. Notably, racial typicality and categorization judgments (averaged across 6 skin color and facial physiognomy combinations) were moderately correlated, $r=.47, p<.001$.

${ }^{4}$ Results for ratings of sadness, anger and happiness are beyond the scope of this paper and are not discussed any further. 
Table 1

Means, Standard Errors for Attractiveness Ratings as a Function of Skin tone and Facial Physiognomy and Results of Pairwise Comparisons within Each Factor in Experiment 2

\begin{tabular}{|c|c|c|c|c|c|c|c|}
\hline \multirow[t]{2}{*}{$\underline{\text { Skin tone }}$} & \multicolumn{7}{|c|}{ Facial } \\
\hline & Mean & Std. Error & $\begin{array}{l}\text { Non-sig. pairwise } \\
\text { comparisons* }\end{array}$ & Physiognomy & Mean & Std. Error & $\begin{array}{l}\text { Non-sig. pairwise } \\
\text { comparisons* }\end{array}$ \\
\hline 1 (dark) & 4.31 & .07 & 1 and 9 & 1 (Afrocentric) & 3.89 & .08 & 1 and 10 \\
\hline 2 & 4.57 & .07 & 2 and $8 ; 2$ and 9 & 2 & 4.30 & .08 & 2 and $9 ; 2$ and 10 \\
\hline 3 & 4.70 & .06 & 3 and $7 ; 3$ and 8 & 3 & 4.63 & .07 & 3 and $8 ; 3$ and 9 \\
\hline 4 & 4.84 & .06 & 4 and $6 ; 4$ and $7 ; 4$ and 8 & 4 & 4.96 & .07 & 4 and $7 ; 4$ and 8 \\
\hline 5 & 4.97 & .06 & 5 and 6 & 5 & 5.19 & .07 & 5 and $6 ; 5$ and 7 \\
\hline 6 & 4.95 & .06 & & 6 & 5.22 & .07 & \\
\hline 7 & 4.82 & .06 & 7 and 8 & 7 & 5.07 & .07 & \\
\hline 8 & 4.71 & .06 & & 8 & 4.71 & .08 & \\
\hline 9 & 4.39 & .07 & & 9 & 4.33 & .09 & \\
\hline 10 (light) & 4.00 & .07 & & 10 (Eurocentric) & 3.96 & .09 & \\
\hline
\end{tabular}

Note. $N=321$. Skin tone levels varied from dark (1) to light (10) and facial physiognomy levels varied from Afrocentric (1) to Eurocentric (10). Racial typicality ratings could range from 1 (Very African American) to 6 (Very Caucasian). *All other pairwise comparisons were significantly different $(p s<.05)$. 
Table 2

Mean Ranks for Skin Tone and Facial Physiognomy in Experiment 2.

\begin{tabular}{cccc}
\hline \multicolumn{2}{c}{ Skin Tone } & & \multicolumn{2}{c}{ Facial Physiognomy } \\
\hline Factor Level & Mean Rank & Factor Level & Mean Rank \\
\hline 1 (Dark) & 1.72 & 1 (Afrocentric) & 1.83 \\
2 & 2.54 & 2 & 2.48 \\
3 & 3.40 & 3 & 3.19 \\
4 & 4.28 & 4 & 4.09 \\
5 & 5.09 & 5 & 5.11 \\
6 & 6.00 & 6 & 6.06 \\
7 & 6.78 & 7 & 7.01 \\
8 & 7.61 & 8 & 7.69 \\
9 & 8.29 & 9 & 8.56 \\
10 (Light) & 9.29 & 10 (Eurocentric) & 8.99 \\
\hline
\end{tabular}




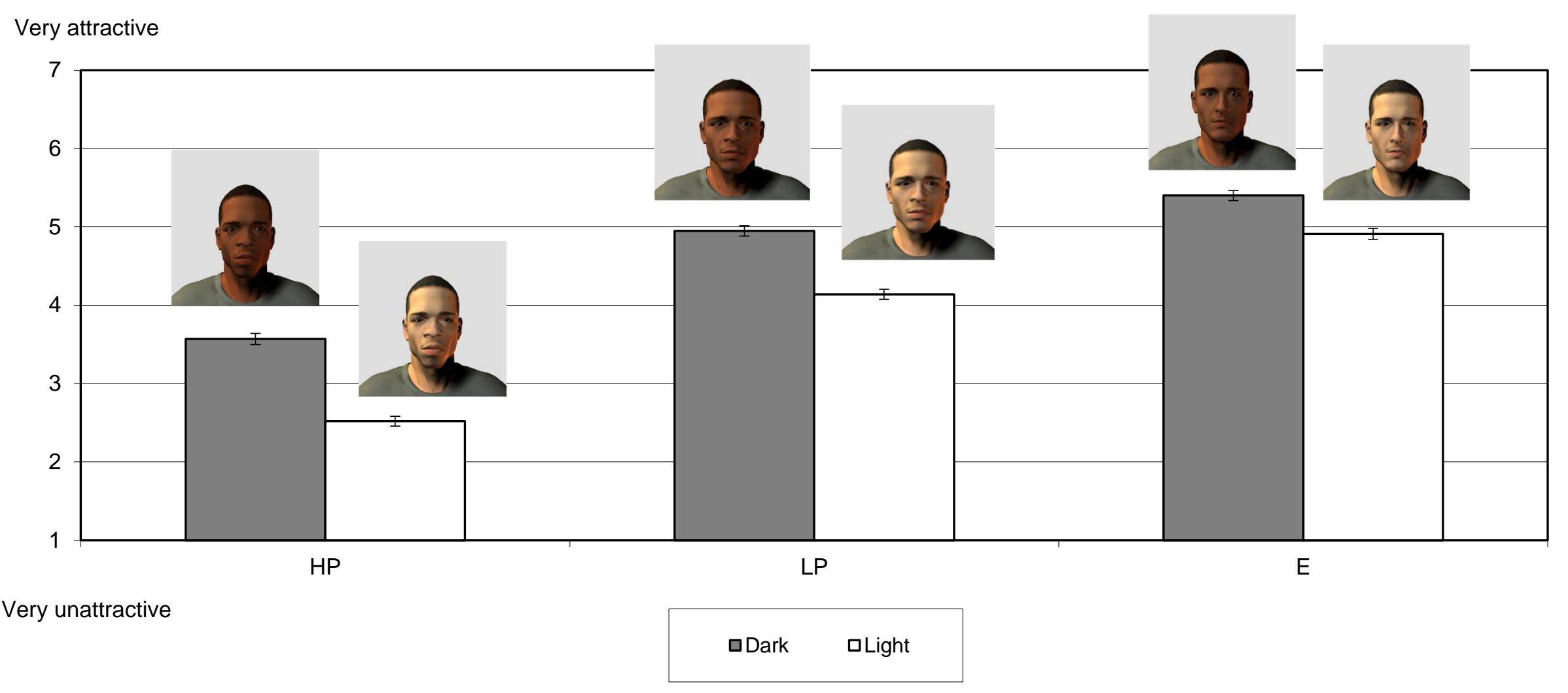

Figure 1. Two-Way Interaction of Physiognomy and Skin tone on Attractiveness Ratings in Experiment 1. HP = high Afrocentric physiognomy; LP = low Afrocentric physiognomy; E = Eurocentric physiognomy. Dark = dark skin tone; Light $=$ light skin tone. Corresponding facial stimuli in polychromatic mode are presented at the top of each bar. Error bars represent standard errors. Facial stimuli are adapted from "Making of a face: Role of facial physiognomy, skin tone, and color presentation mode in evaluations of racial typicality" by E. V. Stepanova \& M. J Strube, 2009, The Journal of Social Psychology, 149, p. 70. Copyright 2009 by Taylor and Francis. Reprinted with permission. 
Very European American

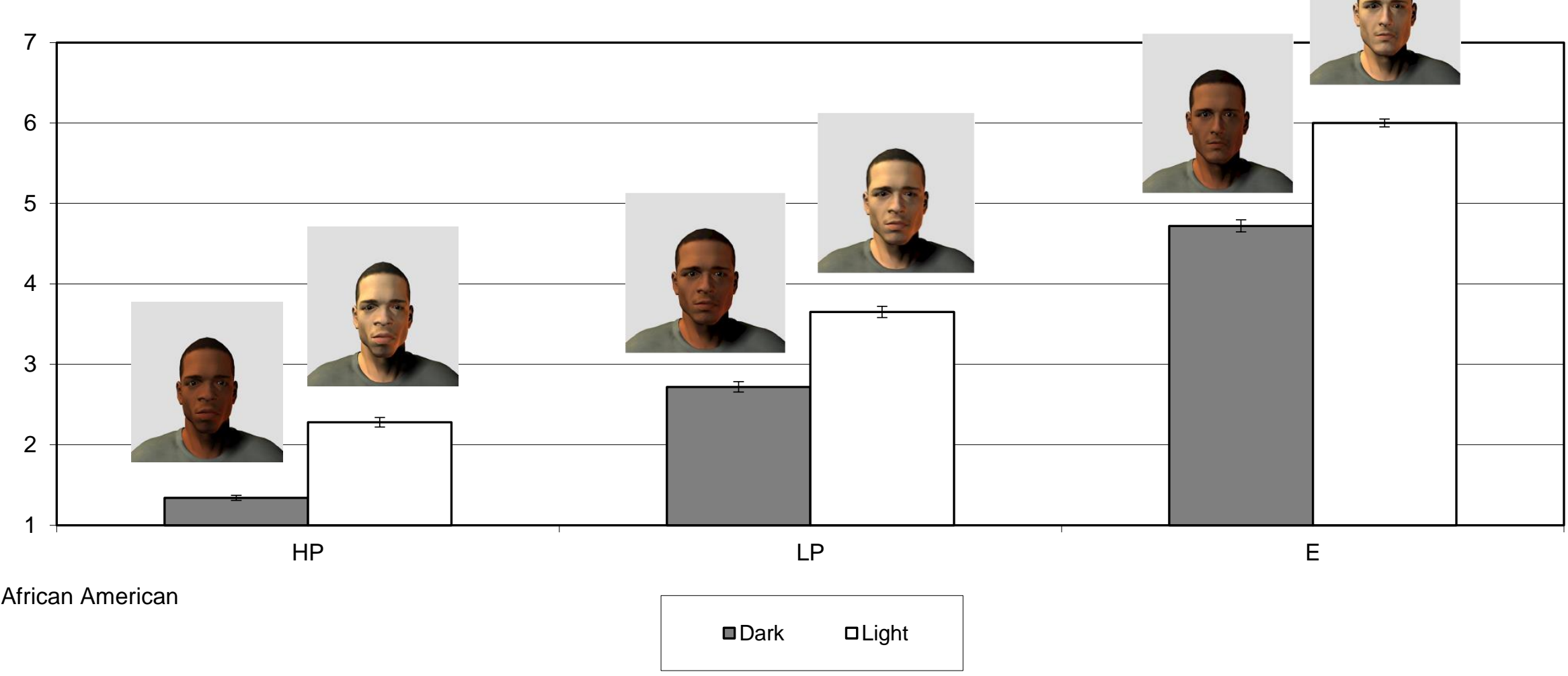

Figure 2. Two-Way Interaction of Physiognomy and Skin tone on Racial Typicality Ratings in Experiment 1 . HP = high Afrocentric physiognomy; LP = low Afrocentric physiognomy; E = Eurocentric physiognomy. Dark = dark skin tone; Light = light skin tone. Corresponding facial stimuli in polychromatic mode are presented at the top of each bar. Error bars represent standard errors. Facial stimuli are adapted from "Making of a face: Role of facial physiognomy, skin tone, and color presentation mode in evaluations of racial typicality” by E. V. Stepanova \& M. J Strube, 2009, The Journal of Social Psychology, 149, p. 70. Copyright 2009 by Taylor and Francis. Reprinted with permission. 




Figure 3. Facial stimuli used in current research, Experiment 2 and 3 (a sample set). Skin tone varies from 1 (dark) to 10 (light), left to right, and physiognomy varies from 1 (Afrocentric) to 10 (Eurocentric), top to bottom. Adapted from "The role of skin tone and facial physiognomy in racial categorization: Moderation by implicit racial attitudes" by E. V. Stepanova and M. J Strube, 2012, Journal Experimental Social Psychology, 48, p. 870. Copyright 2012 by Elsevier. Reprinted with permission. 
Very attractive

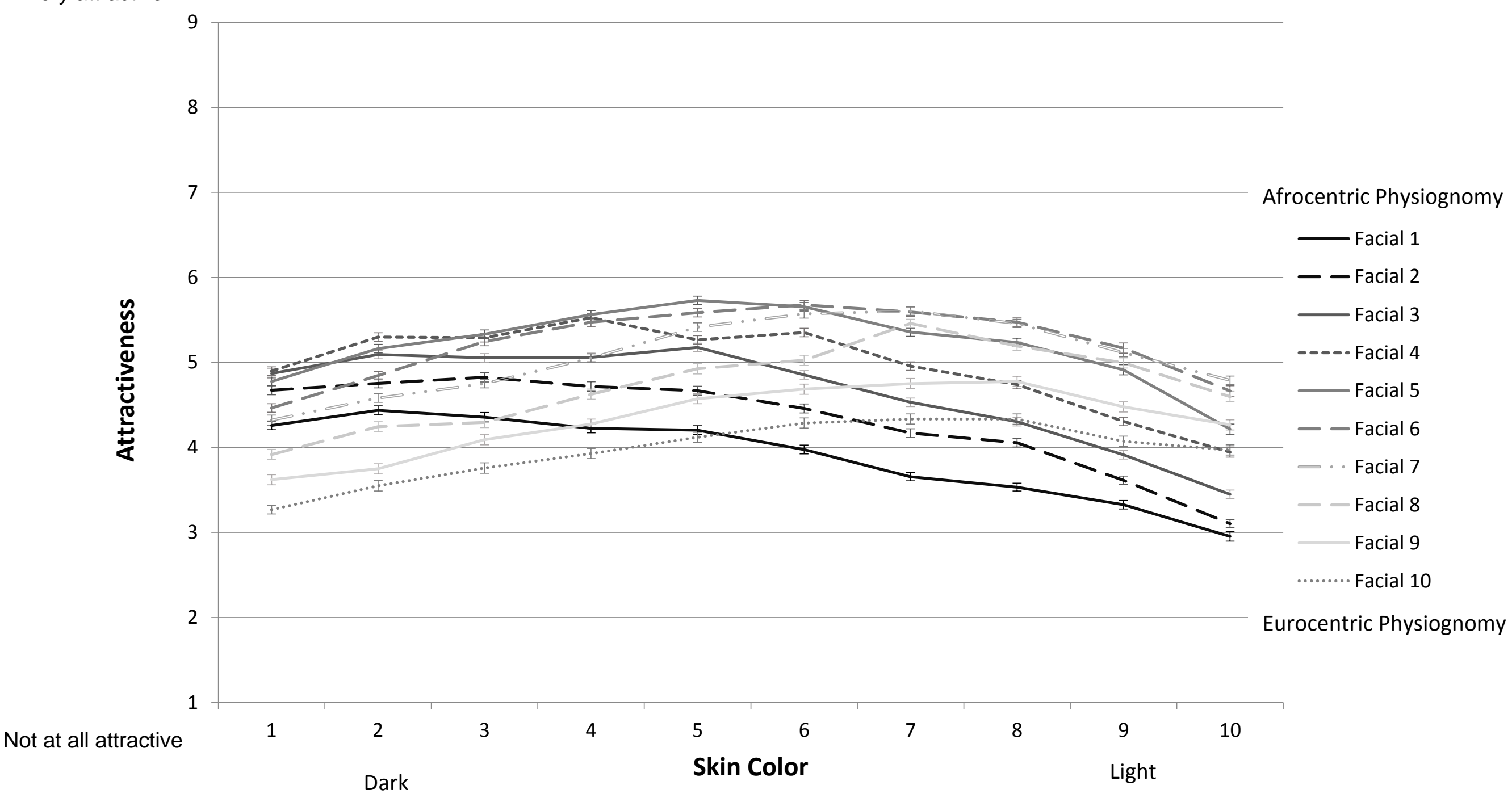

Figure 4. Effects of skin tone and facial physiognomy on attractiveness ratings in Experiment 2. Facial 1= highest Afrocentric physiognomy and facial 10= highest Eurocentric physiognomy. Error bars represent standard errors. 
Very attractive

9

8



3

2

Not at all attractive

1

23

4

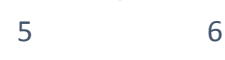

6

8

9

10

\section{Facial Physiognomy}

Afrocentric

Males $\longrightarrow$ Females

Eurocentric

Figure 5. Effects of facial physiognomy and participants' sex on attractivness ratings in Experiment 2. Error bars represent standard errors. 